\title{
Candidate Gene and MicroRNA Biomarkers of Metastatic Renal Cell Carcinoma (MRCC) Response to Sunitinib
}

qiwei yang

XinHua Hospital

wei yang

XinHua Hospital

yijun tian

XinHua Hospital

da Xu

Eastern Hepatobiliary Surgery Hospital

chuanmin chu

Eastern Hepatobiliary Surgery Hospital

xiuwu pan

XinHua Hospital

jianqing ye

XinHua Hospital

jian chu

XinHua Hospital

sishun gan

XinHua Hospital

jianwei cao (D 1063432617@qq.com )

XinHua Hospital

\section{Research Article}

Keywords: metastatic renal cancer, WGCNA, NIPSNAP1 gene, renal cell carcinoma

Posted Date: January 12th, 2022

DOI: https://doi.org/10.21203/rs.3.rs-1228653/v1

License: (c) (1) This work is licensed under a Creative Commons Attribution 4.0 International License.

Read Full License 


\section{Abstract}

Backgrounds: The incidence of renal cancer is relatively insidious, and some patients have been metastatic renal cancer at the initial visit. Sunitinib is the first-line systemic therapy for patients with metastatic renal cell carcinoma, however, there is scant analysis of its effect on genes and microRNAs.

Methods: In this study, 8 differentially expressed microRNAs and 112 differentially expressed genes were designated by analyzing mRNA and microRNA data sets and weighted correlation network analysis (WGCNA).

Results: NIPSNAP1 gene showed the most co-expression with other genes. Through the intersection of the microRNA target gene with our differentially expressed genes, we got 26 genes. KEGG and GO analysis showed that these genes were predominantly concentrated in Pathways in cancer, Sphingolipid metabolism and Glycosaminoglycan degradation. After we set the 26 genes and gene of WGCNA do intersection, received six genes, respectively is NIPSNAP1, SDC4, TBC1D9, NEU1, STK40 and PLAUR.

Conclusion: Through subsequent cell, molecular and flow cytometry experiments, we found the PLAUR would play a crucial role in renal cell carcinoma (RCC) resistant to sunitinib, which will be available for new ideas to forecast sunitinib resistance and reverse sunitinib resistance.

\section{Introduction}

Metastatic Renal Cell Carcinoma (mRCC) is the second only to bladder cancer in the incidence of urinary tract tumors in China, accounting for about $3 \%$ of all malignant tumors ${ }^{1}$.Due to the changes in the living environment and the improvement of diagnostic methods, the incidence of exogenous renal cell carcinoma has increased significantly in recent years, and it has been one of the most important killers threatening human health ${ }^{2}$. The incidence of renal cancer is relatively occult, with $20 \%$ to $30 \%$ of patients having metastatic renal cancer at the first visit ${ }^{3}$, and more than $20 \%$ of patients with localized renal cancer will have metastasis after surgery ${ }^{4}$. The prognosis of metastatic renal cancer is poor, with a median survival of only 13 months and a 5 -year survival rate of less than $10 \%{ }^{5}$. However, due to the effects of occult incidence and limited diagnostic methods of renal cell carcinoma, most of the patients have reached the middle and advanced stage of renal cell carcinoma with varying degrees of metastasis, and are prone to metastasis and recurrence after surgery, resulting in poor prognosis ${ }^{6}$. Cytokine - based nonspecific immunotherapy or combined tumor - reducing nephrectomy was the definitive treatment for metastatic renal cancer ${ }^{7}$. With the development of sequencing technology and bioinformatics, diagnostic and therapeutic potential of miRNA has been $\operatorname{proved}^{8}$. For more than a decade, microRNA and mRNA target gene therapy has become the most promising treatment for metastatic renal cancer in recent years ${ }^{9}$.

MicroRNAs are a class of endogenous non-coding genes in the cells of animals and plants, with a size of about 21-25 bases, which play an important role in the regulation of gene expression ${ }^{10}$. Now studies have 
shown that microRNAs play a regulatory role in tumor development and apoptosis ${ }^{11}$. With further research, microRNAs are expected to become targets and molecular markers for tumor diagnosis, treatment and prognosis ${ }^{12}$. Celia Prior et al. identified the role of mir-942 in drug resistance from a comparison of patients with significant sunitinib efficacy and resistance ${ }^{13}$. Incorvaia Lorena found that a subset of 59 microRNAs involved in mRCC, such as miR-155, miR-22, miR-24, miR-484, miR-335 and miR$492^{14}$. Madrid et al. showed that INF2, Cdc42 and MAL2 could orderly form a pathway regulating endocytosis and lumen formation ${ }^{15}$. These studies suggest that INF2 may be involved in the development and metastasis of renal cancer through its specific intracellular functions. Although there are many studies on MRCC related genes, micro and mRNA studies are still lacking.

Sunitinib is a novel drug that selectively targets a variety of receptor tyrosine kinases. Inhibition of receptor tyrosine kinases is thought to "starve" tumors and have the ability to simultaneously kill tumor cells by blocking the blood and nutrient supply needed for tumor growth. Sunitinib combines antiangiogenesis, which stops the blood supply to tumor cells, with anti-tumor mechanisms that directly attack tumor cells. Sunitinib has also been shown to be active against vegfr, platelet-derived growth factor receptor, stem cell factor receptor, glial cell-derived neurotrophic factor, and fms-like tyrosine kinase- $3^{16}$. Sunitinib, as the standard drug for first-line metastatic renal cell carcinoma, has a better effect than ifn-alfa and improves the production period of patients ${ }^{17}$. First-line observation also found that patients with different metastatic renal cell carcinoma had different tolerance to sunitinib, regardless of age or metastatic site ${ }^{18}$. However, the target genes and micrornas of sunitinib have not been thoroughly studied. The effect, especially in late-stage metastatic renal cell carcinoma, remains mysterious.

In this study, existing microarray expression profile data of metastatic and non-metastatic renal clear cell carcinoma tumor samples were collected from public databases, and bioinformatics methods were used to screen specific differentially expressed genes related to tumor metastasis of renal clear cell carcinoma, and functional annotation and biological function analysis of these genes were performed. At the same time, by analyzing the interactions between proteins encoded by differentially expressed genes, the protein-protein interaction network and the correlation analysis with related small-molecule drugs were constructed to provide certain data support for the clinical diagnosis and drug control treatment of renal clear cell carcinoma.

\section{Results}

\section{Identification of defiantly expression gene and microRNA}

In order to find the differential expression of genes and microRNAs associated with sunitinib treatment, the GSE37766 and GSE65615 datasets were downloaded from NCBI GEO database. Because the mRNA dataset samples were repeated too much, the mRNA differential gene screening criteria were set to $|\log \mathrm{FC}|>0.25$, adjust. $\mathrm{P}<0.05$.The results (Figure 1a) showed that there were 112 differentially expressed genes in sunitinib-treated patients compared with those who did not use sunitinib, including 84 upregulated genes and 28 down-regulated genes. In the same way, a total of 8 microRNAs (Figure 1b) with 
different expressions were found in the microRNA data set ( $|\log F C|>1$, adjust.p<0.05) \consisting of 7 upregulated and 1 down-regulated micoRNA. The 8 differently expressed microRNAs were hsa-miR-30a, hsamiR-30e, hsa-miR-126, hsa-miR-589, hsa-miR-938, hsa-miR-148b, hsa-miR-708 and hsa-miR-18b.

\section{Weighted correlation network analysis of the DEGs associated with sunitinib}

For the identification of sunitinib associated modules and genes, WGCNA was performed on the 112 DEGs. First, we constructed the evolutionary tree of the sample based on the expression data. The samples with excessive missing values were deleted and then analyzed. Next, we filter the power values. Finally, we chose 5 as the value of power. Next, we examined the association between the characteristic values of the network module and metastatic renal cell carcinoma. We got two modules, grey and turquoise. The pine green module contained the most differentially expressed genes, which were selected for further analysis. We used the differentially expressed genes contained in the pine green module to construct the co-expression network, as shown in the figure 1c, which contained 54 edges and 26 points, among which the NIPSNAP1 gene showed the most co-expression characteristics with other genes. GWCNA analysis narrowed the range of target mRNAs and removed the less relevant genes.

\section{KEGG analysis and $\mathrm{GO}$ annotation}

To investigate the biological function of the 26 differentially expressed genes based on the wgcna coexpression network. Gene Ontology (GO) functional and Kyoto Encyclopedia of Genes and Genomes (KEGG) pathway enrichment analysis were performed. GO results (Figure 2a, Table1) showed that囚in level $2 \bigotimes$ these genes mainly play role in binding, catalytic activity and molecular transducer activity. In level 3囚 the functions of these proteins are mainly concentrated in protein binding (20 genes), transferase activity (10 genes) and ion binding (13 genes). For the molecular function part, most genes were enriched in binding, protein binding and catalytic activity which appears in almost all GO analyses. Some interesting molecular functions were also found such as ion binding, cation binding and metal ion binding. For cellular component, most genes belonged to intracellular membrane-bounded organelle, cytoplasmic part and membrane-bounded vesicle. For the biological process, 16 genes were associated with metabolic process and 15 genes were associated with biological regulation. The results of KEGG (Figure 2b) showed that the main enrichment pathways of the 26 genes are as follows囚Pathways in cancer (PTGER3, LAMA3 and TXNRD), Sphingolipid metabolism (NEU1 and GLB1) and Glycosaminoglycan degradation (hya and GLB1).

\section{MicroRNA target gene prediction}

Using miRTarBase, the microRNA-target interacted gene which was experimentally validated were selected based on the DEG microRNAs. Totally, 162 genes were associated with differentially expressed microRNAs. To narrow the range, we cross-referenced the differentially expressed genes with all mRNAtarget genes (Table 2). The results showed that a total of $26(0.9 \%)$ genes were present simultaneously in both gene sets such as NIPSNAP1, CDK6, PPARA, FGFR1 and CDR1. After that, the 26 genes were intersected with the genes in WGCNA. As shown in the figure 2c, a total of 6 genes were found in the 
WGCNA and the differential microRNA target gene. These genes are NIPSNAP1, SDC4, TBC1D9, NEU1, STK40 and PLAUR.

\section{PCA and PCOA}

To test the representativeness of the differential genes, principal component analysis (PCA) and principal co-ordinates analysis (PCOA) were finished based on the 112 differently expressed genes. PCA is a dimension reduction algorithm commonly used in data mining. PCA uses the idea of dimensionality reduction to reduce the dimensionality of high-dimensional variables in the principle of minimizing data loss, that is, to find a few comprehensive indexes among numerous variables. PCoA is a visualization method to study the similarity or difference of data. After sorting through a series of eigenvalues and eigenvectors, the principal ordinates are selected. The PCA result (Figure 2d) showed that two groups with (red circle) or without (green circle) sunitinib clearly separated from each other. The first principal component and the second principal component explained $31.2 \%$ and $7.9 \%$ of the total variation, respectively. PCoA results (Figure $2 \mathrm{e}$ ) show similar results with PCA results that the first primary coordinate and the second primary coordinate explained the $34.04 \%$ and $11.98 \%$ of the total variation, respectively. Combining the results of PCA and PCOA, the 112 differently expressed genes fully reflect the therapeutic effect of sunitinib on metastatic renal cell carcinoma.

\section{The upregulated six genes in sunitinib resistant RCC cells}

In order to confirm the NIPSNAP1, SDC4, TBC1D9, NEU1, STK40 and PLAUR expression, we grafted sunitinib resistant 7860 (SR7860) cells and 7860 cells (sunitinib sensitive) into nude mice forming tumor, and the qRT-PCR experiments were performed and the results showed in the figure 3 . It indicated that in the six hub genes, only PLAUR were obviously high expressed in sunitinib resistant RCC tissues compared with sensitive RCC tissues, which were paritially in accordance with the previous data acquired from the bioinformatics analysis.

In order to verify the role of NIPSNAP1, SDC4, TBC1D9, NEU1, STK40 and PLAUR in the regulation of sunitinib-resistance in sunitinib-resistance RCC cells, we interfere them with siRNA-1 and siRNA-2 in sunitinib-resistance 7860 (SR7860) cells. The qRT-PCR result showed that siRNA-1/siRNA-2 obviously decrseased NIPSNAP1, SDC4, TBC1D9, NEU1, STK40 and PLAUR levels in the trancfected SR7860 cells (figure. 4a). So we test the cellular functions of down regulation of NIPSNAP1, SDC4, TBC1D9, NEU1, STK40 and PLAUR. Evidently, interference of PLAUR inhibited sunitinib resistance were most effectively in contrast to NIPSNAP1, SDC4, TBC1D9, NEU1 and STK40 (figure 4b).

\section{PLAUR regulates sunitinib resistance in RCC cells}

To further demonstrate the function of PLAUR in sunitinib-resistant RCC cells, we steady knocked down PLAUR by transfecting lentiviral-mediated short hairpin RNA (shRNA) in SR7860 cells (figure 5a). We cultivated SR7860 cells, which were transfected with negative shRNA or PLAUR shRNA-1/-2 
A morphological detection of the incubated cells with $2 \mu \mathrm{mol}$ sunitinib for 60 hours, which manifested that PLAUR knocked down cells changed significantly from fusiform shape to round shape, and easier to float compared with negative control (figure 5c). In order to confirm this phenomenon, the CCK8 experiment was conducted and the result showed that both shPLAUR-1 and shPLAUR-2 remarkably suppressed the proliferation of SR7860 cells in contrast to the negative control upon sunitinib administration at indicated concentrations for 60 hours (figure $5 \mathrm{~b}$ ). To verify the therapeutic action of PLAUR knockdown on sunitinib resistance in SR7860 cells, the analysis of SR7860 cells by flow cytometry and annexin V-FITC/PI double dyeing experiment were conducted. The proportion of apoptosis in early and late were obviously elevated in PLAUR downregultaed SR7860 cells in contrast to the negative control when treated with $2 \mu \mathrm{M}$ sunitinib (figure $6 \mathrm{a}$ and $\mathrm{b}$ ). In addition, the cell cycle experiment were performed to detect outcome of the PLAUR knockdown in sunitinib resistant RCC cells. The result suggested the percentage of SR7860 cells in G2 phase clearly increased relative to negative control (figure $6 \mathrm{c}$ and $\mathrm{d}$ ).

So the apoptosis signaling antibody kit were used in the western blot experiment to test the levels of apoptosis relevant protein (figure 7a). The result suggested that the expression of cleaved caspase-3, cleaved caspase- 9 and cleaved PARP were remarkably upregulated in SR7860 cells transfected with shPLAUR-1 and shPLAUR-2 as opposed to negative control (figure $7 \mathrm{~b}, \mathrm{c}, \mathrm{d}$ and e).

\section{Discussion}

A growing number of researchers are concerned about the role of microRNAs in cancer development because they regulate gene expression and ultimately alter cell survival cycles. For instance $\ m i R-18 b$, hsa-miR-30a囚hsa-miR-30e which were included in mir-17-92 cluster were dysregulated in ccRCC in a study by Chow, Tsz-fung F. et $\mathrm{al}^{33}$. However, information on microRNAs as markers of sunitinib tolerance to MRCC is limited. We found eight differentially expressed microRNAs by comparing sunitinib-sensitive patients with drug resistance. They were hsa-mir-30a, hsa-mir-30e, hsa-mir-126, hsa-mir-589, hsa-mir-938, hsa-mir-148b, hsa-mir-708 and hsa-mir-18b, respectively. This is partly consistent with a previous study which found that hsa-miR-18 involved in resistance to sunitinib in Renal Cell Carcinoma Cells ${ }^{34}$. GámezPozo et al also reported that twenty-eight microRNAs of the 287 include the has-miR-126 were related to resistance to first-line sunitinib in advanced renal cell carcinoma patients ${ }^{35}$. However, in another study, candidate MicroRNA biomarkers in response to sunitinib therapy in metastatic renal cell carcinoma were not the same as in this study ${ }^{4}$ (miR-155, miR-484, miR-221, miR-222, miR-425, miR-133, miR-410, miR141, miR-628 and miR-942). To sum up, the differential expression of microRNA in MRCC patients to some extent reflects the difference in response to sunitinib drug treatment. In any case, more detailed experiments are needed to demonstrate the role of mRNAs in sunitinib resistance.

Sunitinib resistance is a challenge in advanced renal cell carcinoma. Therefore, it is necessary to understand the underlying mechanisms of sunitinib resistance. We compared patients with or without different responses to sunitinib and mined 26 potential hub genes through WGCNA analysis. These 26 hub genes were then intersected with microRNA target genes to obtain 6 genes in total. These six genes 
are NIPSNAP1, SDC4, TBC1D9, NEU1, STK40, PLAUR. Based on previous studies『NIPSNAP1 belong to a highly conservative family of proteins of unknown function which is mainly associated with cognitive deficits $^{36}$, pain transmission ${ }^{37}$, and neurological dysfunction ${ }^{38}$. Studies have shown that the NIPSNAP1 gene is inactivated during malignant metastasis of prostatic epithelial cells ${ }^{39}$.NIPSNAP1 was identified as a tumor suppressor in prostate cancer in a previous study identified as a tumor suppressor in prostate cancer $^{40}$. We hypothesized that sunitinib might activate the NIPSNAP1 gene in some people to activate anti-tumor immune protein activity. Much work remains to be done. Syndecan-4 (SDC4) SDC4 plays an important role in the development and metastasis of renal cell carcinoma ${ }^{11}$. The decrease of tumor metastasis and necrosis will lead to the upregulation of SDC4 41,43 . In ros 1 positive lung cancer, an activating KIT mutation of SDC4 induces crizotinib resistance ${ }^{42}$. We suspect that sunitinib may also cause mutations in SDC4 that lead to changes in resistance.

MicroRNA-125b was reported to promote invasion and metastasis of gastric cancer by targeting NEU1 ${ }^{44}$. No significant changes in microrna-125b were found in our results, and sunitinib may not affect microrna125b. Plasminogen activator receptor (PLAUR) has long been associated with human colon cancer, and abnormalities in the foxm1-PLAUR signaling pathway play a key role in the progression and metastasis of human colon cancer ${ }^{45}$. The overexpression of PLAUR and SERPINE1 has been correlated with poor survival outcomes and a high-grade tumor in $\mathrm{RCC}^{46}$. Our results also implied the association between PLAUR and metastatic renal cell carcinoma. Only in our results, the microRNAs related to PLAUR were hsa-mir-622 and hsa-mir-335 which had no significant difference in expression.

We found PLAUR were obviously upregulated in sunitinib resistant RCC cells in contrast to sunitinib sensitive RCC cell tissues. While, downregulation of PLAUR make the sunitinib resistant RCC cells acquired better response to sunitinib compared to the other five genes in the loss-of-function analysis. Previous studies have reported the PLAUR induces non-small-cell lung cancer acquired gefitinibresistance through EGFR/p-AKT/survivin signaling pathway ${ }^{47}$. In our study, knockdown of PLAUR in sunitinib resistant RCC cells promoted apoptosis in the sunitinib treatment and upregulated the expression of cleaved caspase-3, caspase-9 and PARP in contrast to the negative control.

\section{Conclusion}

A total of 112 differentially expressed genes and 8 microRNAs were found in two-group (with or without sunitinib) comparisons of patients with metastatic renal cell carcinoma. Weighted correlation network analysis (WGCNA) results showed that NIPSNAP1 gene showed the most co-expression with other genes. Through the intersection of the microRNA target gene with our differentially expressed genes, we got 26 genes. KEGG and GO analysis showed that these genes were mainly concentrated in Pathways in cancer, Sphingolipid metabolism and Glycosaminoglycan degradation. After we put the 26 genes and gene of WGCNA do intersection, received six genes, respectively is NIPSNAP1, SDC4, TBC1D9, NEU1, STK40 and PLAUR. The PLAUR may induce renal cell carcinoma resistance to sunitinib. Postoperative specimens 
and serum PLAUR levels may serve as a biomarker to forecast the RCC patients' response to sunitinib, and inhibiting PLAUR may help sunitinib resistant RCC patients acquired better response to sunitinib.

\section{Materials And Methods}

\section{Datasets}

We obtained mRNAs and microRNAs expression date of Metastatic Renal Cell Carcinoma (mRCC) from the NCBI GEO database ${ }^{19}$ (https://www.ncbi.nlm.nih.gov/). We selected two datasets including GSE65615 (47 samples without sunitinib treatment,75 samples with sunitinib treatment) as mRNA dataset, GSE37766 ( 3 samples sensitive to sunitinib, 3 samples resistance to sunitinib) as microRNA dataset.

The mRNA and miRNA combined datasets for myocardial infarction analysis were extracted from the Series Matrix File, respectively. The probe ID was converted into gene symbol or miRNA ID through the platform annotation information table. The same gene symbol or miRNA ID was incorporated. Expression matrixes of mRNA and miRNA samples were screened out according to ID. Other data sets are preprocessed in the same way.

\section{Identification of DEGs}

The identification of differentially expressed genes (DEGs) were performed with R software $33.4 .3 \rrbracket$ ${ }^{20}$, ,edgeR (version 3.5.2) ${ }^{21}$ \http://bioconductor.org/packages/release/bioc/html/edgeR.html囚. The t-test method was used to test the difference between two groups. After t-test, all the genes obtained corresponding p-values. In order to make the results more reliable, we conducted multiple tests to correct the p-values through the Benjamin and Hochberg method. Finally, the threshold of DEGs was set as $|\log F C|>0.25$, adjust.p $<0.05$. The obtained differential gene counts values were used for volcano plot and heat map.

\section{Functional analysis of DEGs}

In order to further clarify the functional annotation of DEGs, The common enrichment analysis tools AGRIgo $^{22}$ (http://systemsbiology.cau.edu.cn/agriGov2/index.php) and DAVID ${ }^{23} \rrbracket$ version $\llbracket 6.8 \rrbracket$ https://david.ncifcrf.gov/『were used to GO enrichment analysis of Gene Ontolog BP function and explore KEGG pathways analysis, respectively. The cutoff criterion was $P-V a l u e<0.05$ and count $>1$. Protein sequences were extracted from the human genome protein bank based on the gene symbol using our own Perl scripts. We performed separate notes of DEGs using kaas ${ }^{24}$ (https://www.genome.jp/tools/kaas/).

\section{Construction of the WGCNA co-expression network}


$R \rrbracket 3.5 .0 \rrbracket p a c k a g e$ WGCNA ${ }^{25}$ (1.6.6) was used to build the co-expression network. First of all, we need to process our data, the screening criteria were as follows: (a) retain $75 \%$ of the genes with higher absolute deviation, (b) the MAD value was greater than 0.01 . Construct a sample tree to check whether or not outliers exist and determine the soft threshold (power value). Construct a hierarchical clustering tree. to show each module, and draw the correlation graph of each module. Visual gene network (TOM plot) was constructed, the nodes of the top 100 were screened according to the wight value between nodes, and the network was exported and plotted by Cytoscape ${ }^{26}$. In detail, no outlier samples were found in this study. The soft threshold (power value) was 6 for the following analysis.

\section{MicroRNA target gene prediction}

MicroRNA target genes that were supported by strong experimental evidences (Reporter assay or Western blot) were downloaded from mirTarbase ${ }^{27}$ (http://mirtarbase.mbc.nctu.edu.tw/php/download.php). Inhouse perl scripts were used to extract the data information and remove the irrelevant data based on the differently expressed microRNAs.

\section{Principal component analysis (PCA) and principal co-ordinates analysis (PCoA)}

An in-house perl scripts were used to obtain the expression data of the differently expressed gene and the grouping information. Then $\mathrm{R}$ (version 3.5.0) packages ggplot $2^{28}$, plyr $^{29}$, scales ${ }^{30}$, and ggbiplot ${ }^{31}$ were applied together to do the principal component analysis. The $\mathrm{X}$-axis represents the percentage of the first principal component to the total variation. The $Y$-axis represents the percentage of the second principal component in the total variation. Principal co-ordinates analysis was completed by $\mathrm{R}$ (version 3.5.0) ggplot2 and vegan ${ }^{32}$ with the hellinger method based on the expression data and grouping information.

\section{Cells and cell culture}

The human renal cell carcinoma cell line 7860 was purchased from the American Type Culture Collection (ATCC, Rockville, MD, USA). In order to cultivate sunitinib-resistant 7860 cells, we successively incubated sunitinib-sensitive 7860 cells to an initial dosage of sunitinib $(2 \mu \mathrm{M})$ and consecutively incremental concentrations up to $5 \mu \mathrm{M}$. The sunitinib-sensitive 7860 and -resistant 7860 cells were maintained in RPMI 1640 medium, which were supplemented with $10 \% \mathrm{FBS}, 100 \mathrm{IJ} / \mathrm{ml}$ penicillin and $100 \mu \mathrm{g} / \mathrm{ml}$ (Thermo Fisher Scientific, MA, USA).

\section{Cell counting kit-8 (CCK-8) experiment}

SR7860 cells were seeded into 96-well plates at the approximately 1000 cells per well with $200 \mu \mathrm{l}$ of culture medium. 24 hours late, $10 \mu$ l CCK8 reagents (Dojindo laboratories, Japan) were added into each well, and the plates were incubated for 2 hours at $37^{\circ} \mathrm{C}$. Finally the optical density were detected at the wavelength of $450 \mathrm{~nm}$.

\section{Flow cytometry of cell apoptosis}


Cell apoptosis was evaluated using the Annexin V-FITC/PI Apoptosis Detection Kit according to the explanatory memorandom, and all steps were carried out on ice to keep a $4{ }^{\circ} \mathrm{C}$ temperature. The transfected SR7860 were treated with $5 \mu \mathrm{M}$ sunitinib, washed with PBS and re-suspended in FITC Annexin V binding buffer, followed by incubated with Annexin V-FITC and stained with PI (Thermo Fisher Scientific). In the end the proportion of apoptotic cells was assessed through flow cytometry.

\section{Flow cytometry cell cycle experiment}

The transfected SR7860 cells were seeded in 6-well plates $\left(5 \times 10^{5}\right.$ cells/well) and were incubated with $5 \mu \mathrm{M}$ sunitinib. Subsequently, the SR7860 cells were washed with PBS and suspended in DNA staining solution added with $5 \mu$ l of permeabilization solution for half an hour at normal temperature. In the end, the proportion of cells in the cell cycle phase was evaluated on the SONY SH800 Cell Sorter (Tokyo, Japan).

\section{Quantitative reverse transcription PCR}

Total RNA was isolated using TRIzol Reagent (Thermo Fisher Scientific, MA, USA), and cDNA was synthesized using reverse transcription kit (Tadara Biotechnology Co., Dalian, China). Afterwards, qRTPCR was carried out using the ABI StepOnePlus system (Thermo Fisher Scientific, Inc.). Relative expression levels was computed through $-2 \Delta \triangle \mathrm{Ct}$ method, and the $\beta$-actin was served as internal control. PLAUR primer sequences were sense 5'- GCTTGTGGGAAGAAGGAGAA-3', and the anti-sense 5'CCTCGGTAAGGCTGGTGAT-3'. The qRT-PCR experiment was repeated at least 3 times in triplicate.

\section{Western blot experiment}

RIPA kit (Beyotime Biotechnology, China) and phosphorylation protease inhibitor were used to abstract the protein of cells. The proteins were separated by gel electrophoresis (SDS-PAGE) and then transferred to PVDF membranes (Millipore, Bedford, MA, USA). The membranes were incubated with anti-PLAUR (cat. no. \#12863, 65kDa, CST, USA), Cleaved caspase-3 (cat. no. \#9553, 17 kDa, CST, USA), Cleaved caspase-9 (cat. no. \#20750, 37kDa, CST, USA), Cleaved PARP (cat. no. \#5625, 89kDa, CST, USA), $\beta$-actin (cat. no. $\# 3700,45 \mathrm{kDa}, \mathrm{CST}, \mathrm{USA}$ ) antibodies at $4^{\circ} \mathrm{C}$ overnight. The next day, the membranes were washed and incubated with secondary antibodies at room temperature for 1.5 hours. Finally, the protein expression levels were detected via Western Blot Detection System (Amersham Imager 600).

\section{Datasets}

We obtained mRNAs and microRNAs expression date of Metastatic Renal Cell Carcinoma (mRCC) from the NCBI GEO database ${ }^{19}$ (https://www.ncbi.nlm.nih.gov/). We selected two datasets including GSE65615 (47 samples without sunitinib treatment,75 samples with sunitinib treatment) as mRNA dataset, GSE37766 ( 3 samples sensitive to sunitinib, 3 samples resistance to sunitinib) as microRNA dataset. 
The mRNA and miRNA combined datasets for myocardial infarction analysis were extracted from the Series Matrix File, respectively. The probe ID was converted into gene symbol or miRNA ID through the platform annotation information table. The same gene symbol or miRNA ID was incorporated. Expression matrixes of mRNA and miRNA samples were screened out according to ID. Other data sets are preprocessed in the same way.

\section{Identification of DEGs}

The identification of differentially expressed genes (DEGs) were performed with R software $₫ 3.4 .3 \rrbracket$

20, ,edgeR (version 3.5.2) ${ }^{21}$ 凶http://bioconductor.org/packages/release/bioc/html/edgeR.html冈. The t-test method was used to test the difference between two groups. After t-test, all the genes obtained corresponding $p$-values. In order to make the results more reliable, we conducted multiple tests to correct the $p$-values through the Benjamin and Hochberg method. Finally, the threshold of DEGs was set as $|\log F \mathrm{C}|>0.25$, adjust. $p<0.05$. The obtained differential gene counts values were used for volcano plot and heat map.

\section{Functional analysis of DEGs}

In order to further clarify the functional annotation of DEGs, The common enrichment analysis tools AGRIgo ${ }^{22}$ (http://systemsbiology.cau.edu.cn/agriGOv2/index.php) and DAVID ${ }^{23} \rrbracket$ version $₫ 6.8 \rrbracket$

https://david.ncifcrf.gov/『were used to GO enrichment analysis of Gene Ontolog BP function and explore KEGG pathways analysis, respectively. The cutoff criterion was $P$-Value $<0.05$ and count $>1$. Protein sequences were extracted from the human genome protein bank based on the gene symbol using our own Perl scripts. We performed separate notes of DEGs using kaas ${ }^{24}$

(https://www.genome.jp/tools/kaas/).

\section{Construction of the WGCNA co-expression network}

$\mathrm{R} 囚$ 3.5.0囚package WGCNA ${ }^{25}$ (1.6.6) was used to build the co-expression network. First of all, we need to process our data, the screening criteria were as follows: (a) retain $75 \%$ of the genes with higher absolute deviation, (b) the MAD value was greater than 0.01. Construct a sample tree to check whether or not outliers exist and determine the soft threshold (power value). Construct a hierarchical clustering tree. to show each module, and draw the correlation graph of each module. Visual gene network (TOM plot) was constructed, the nodes of the top 100 were screened according to the wight value between nodes, and the network was exported and plotted by Cytoscape ${ }^{26}$. In detail, no outlier samples were found in this study. The soft threshold (power value) was 6 for the following analysis.

\section{MicroRNA target gene prediction}

MicroRNA target genes that were supported by strong experimental evidences (Reporter assay or Western blot) were downloaded from mirTarbase ${ }^{27}$ (http://mirtarbase.mbc.nctu.edu.tw/php/download.php). In- 
house perl scripts were used to extract the data information and remove the irrelevant data based on the differently expressed microRNAs.

\section{Principal component analysis (PCA) and principal co-ordinates analysis (PCOA)}

An in-house perl scripts were used to obtain the expression data of the differently expressed gene and the grouping information. Then $\mathrm{R}$ (version 3.5.0) packages ggplot $2^{28}$, plyr $^{29}$, scales ${ }^{30}$, and ggbiplot ${ }^{31}$ were applied together to do the principal component analysis. The X-axis represents the percentage of the first principal component to the total variation. The $Y$-axis represents the percentage of the second principal component in the total variation. Principal co-ordinates analysis was completed by $\mathrm{R}$ (version 3.5.0) ggplot2 and vegan ${ }^{32}$ with the hellinger method based on the expression data and grouping information.

\section{Cells and cell culture}

The human renal cell carcinoma cell line 7860 was purchased from the American Type Culture Collection (ATCC, Rockville, MD, USA). In order to cultivate sunitinib-resistant 7860 cells, we successively incubated sunitinib-sensitive 7860 cells to an initial dosage of sunitinib $(2 \mu \mathrm{M})$ and consecutively incremental concentrations up to $5 \mu \mathrm{M}$. The sunitinib-sensitive 7860 and -resistant 7860 cells were maintained in RPMI 1640 medium, which were supplemented with $10 \% \mathrm{FBS}, 100 \mathrm{IU} / \mathrm{ml}$ penicillin and $100 \mu \mathrm{g} / \mathrm{ml}$ (Thermo Fisher Scientific, MA, USA).

\section{Cell counting kit-8 (CCK-8) experiment}

SR7860 cells were seeded into 96-well plates at the approximately 1000 cells per well with $200 \mu \mathrm{l}$ of culture medium. 24 hours late, $10 \mu$ l CCK8 reagents (Dojindo laboratories, Japan) were added into each well, and the plates were incubated for 2 hours at $37^{\circ} \mathrm{C}$. Finally the optical density were detected at the wavelength of $450 \mathrm{~nm}$.

\section{Flow cytometry of cell apoptosis}

Cell apoptosis was evaluated using the Annexin V-FITC/PI Apoptosis Detection Kit according to the explanatory memorandom, and all steps were carried out on ice to keep a $4{ }^{\circ} \mathrm{C}$ temperature. The transfected SR7860 were treated with $5 \mu \mathrm{M}$ sunitinib, washed with PBS and re-suspended in FITC Annexin V binding buffer, followed by incubated with Annexin V-FITC and stained with PI (Thermo Fisher Scientific). In the end the proportion of apoptotic cells was assessed through flow cytometry.

\section{Flow cytometry cell cycle experiment}

The transfected SR7860 cells were seeded in 6-well plates $\left(5 \times 10^{5}\right.$ cells/well) and were incubated with $5 \mu \mathrm{M}$ sunitinib. Subsequently, the SR7860 cells were washed with PBS and suspended in DNA staining solution added with $5 \mu$ l of permeabilization solution for half an hour at normal temperature. In the end, the proportion of cells in the cell cycle phase was evaluated on the SONY SH800 Cell Sorter (Tokyo, Japan). 


\section{Quantitative reverse transcription PCR}

Total RNA was isolated using TRIzol Reagent (Thermo Fisher Scientific, MA, USA), and cDNA was synthesized using reverse transcription kit (Tadara Biotechnology Co., Dalian, China). Afterwards, qRTPCR was carried out using the ABI StepOnePlus system (Thermo Fisher Scientific, Inc.). Relative expression levels was computed through $-2 \Delta \triangle \mathrm{Ct}$ method, and the $\beta$-actin was served as internal control. PLAUR primer sequences were sense 5'- GCTTGTGGGAAGAAGGAGAA-3', and the anti-sense 5'CCTCGGTAAGGCTGGTGAT-3'. The qRT-PCR experiment was repeated at least 3 times in triplicate.

\section{Western blot experiment}

RIPA kit (Beyotime Biotechnology, China) and phosphorylation protease inhibitor were used to abstract the protein of cells. The proteins were separated by gel electrophoresis (SDS-PAGE) and then transferred to PVDF membranes (Millipore, Bedford, MA, USA). The membranes were incubated with anti-PLAUR (cat. no. \#12863, 65kDa, CST, USA), Cleaved caspase-3 (cat. no. \#9553, 17 kDa, CST, USA), Cleaved caspase-9 (cat. no. \#20750, 37kDa, CST, USA), Cleaved PARP (cat. no. \#5625, 89kDa, CST, USA), $\beta$-actin (cat. no. $\# 3700,45 \mathrm{kDa}, \mathrm{CST}, \mathrm{USA}$ ) antibodies at $4^{\circ} \mathrm{C}$ overnight. The next day, the membranes were washed and incubated with secondary antibodies at room temperature for 1.5 hours. Finally, the protein expression levels were detected via Western Blot Detection System (Amersham Imager 600).

\section{Abbreviations}

\begin{tabular}{|ll|}
\hline Abbreviation & Complete spelling \\
\hline mRCC & Metastatic Renal Cell Carcinoma \\
\hline WGCNA & Weighted correlation network analysis \\
\hline GO & Gene Ontology \\
\hline CC & cellular component \\
\hline MF & molecular function \\
\hline BP & biological process \\
\hline KEGG & Kyoto Encyclopedia of Genes and Genomes \\
\hline NCBI & National Center for Biotechnology Information \\
\hline DEG & differentially expressed genes \\
\hline PCA & principal component analysis \\
\hline PCoA & principal co-ordinates analysis \\
\hline qRT-PCR & Quantitative Real-time PCR \\
\hline
\end{tabular}




\section{Declarations}

\section{Consent for publication}

Not applicable.

\section{Availability of data and materials}

The datasets used and/or analysed in the present study are available from the corresponding author based on legitimate needs and purposes.

\section{Competing interests}

All authors declare no competing financial interest.

\section{Funding}

Sponsored by National Natural Science Foundation of China for Youths (No. 8200100057), Shanghai jiading district health commission scientific research project youth fund (No. 2020-QN-02), Shanghai Sail Program of Young Talents of Science and Technology (No. 19YF1459700). Meng Chao Talent Training Plan of Eastern Hepatobiliary Surgery Hospital.

\section{Authors' contributions}

YQW had wrote the original draft. XD and CCM had finished the data collection and curation. YW and TYJ had accomplished the data and formal analysis. YJQ and PXW had designed the experiment and participated the conceptulization. CJ and GSS had take part in the writing review and editing. CJW had supervised all the study. All authors reviewed the manuscript.

\section{References}

1. Song, Wenbin, et al. "Targeting newly identified ERB/TGF- $\beta 1 /$ SMAD3 signals with the FDA-approved anti-estrogen Faslodex or an ER $\beta$ selective antagonist in renal cell carcinoma." Molecular oncology 12.12 (2018): 2055-2071.

2. Gupta, Kiran, et al. "Epidemiologic and socioeconomic burden of metastatic renal cell carcinoma (mRCC): a literature review." Cancer treatment reviews 34.3 (2008): 193-205.

3. Naito, Sei, et al. "Prognosis of Japanese metastatic renal cell carcinoma patients in the cytokine era: a cooperative group report of 1463 patients." European urology 57.2 (2010): 317-326.

4. Kovacova, Julia, et al. "Candidate microRNA biomarkers of therapeutic response to sunitinib in metastatic renal cell carcinoma: A validation study in patients with extremely good and poor response." Anticancer research 38.5 (2018): 2961-2965.

5. Powles, Thomas, et al. "Updated European Association of Urology Guidelines: recommendations for the treatment of first-line metastatic clear cell renal cancer." European urology 73.3 (2018): 311-315. 
6. Gupta, Kiran, et al. "Epidemiologic and socioeconomic burden of metastatic renal cell carcinoma (mRCC): a literature review." Cancer treatment reviews 34.3 (2008): 193-205.

7. Gouttefangeas, Cécile, et al. "Immunotherapy of renal cell carcinoma." Cancer Immunology, Immunotherapy 56.1 (2007): 117-128.

8. Borel, Florie, Pavlina Konstantinova, and Peter LM Jansen. "Diagnostic and therapeutic potential of miRNA signatures in patients with hepatocellular carcinoma." Journal of hepatology 56.6 (2012): 1371-1383.

9. Grassi, Paolo, et al. "Cabozantinib in the treatment of advanced renal cell carcinoma: design, development, and potential place in the therapy." Drug design, development and therapy 10 (2016): 2167.

10. Reinhart, Brenda J., et al. "MicroRNAs in plants." Genes \& development 16.13 (2002): 1616-1626.

11. Liu, Can, and Dean G. Tang. "MicroRNA regulation of cancer stem cells." Cancer research 71.18 (2011): 5950-5954.

12. Taylor, Douglas D., and Cicek Gercel-Taylor. "MicroRNA signatures of tumor-derived exosomes as diagnostic biomarkers of ovarian cancer." Gynecologic oncology 110.1 (2008): 13-21.

13. Prior, Celia, et al. "Identification of tissue microRNAs predictive of sunitinib activity in patients with metastatic renal cell carcinoma." PloS one 9.1 (2014): e86263.

14. Incorvaia, Lorena, et al. "A novel predictive biomarker of immunotherapy response in metastatic renal cell carcinoma (mRCC): The lymphocyte microRNA expression profile." (2019): e16109-e16109.

15. Madrid, Ricardo, et al. "The formin INF2 regulates basolateral-to-apical transcytosis and lumen formation in association with Cdc42 and MAL2." Developmental cell 18.5 (2010): 814-827.

16. Kulke, Matthew H., et al. "Activity of sunitinib in patients with advanced neuroendocrine tumors." Journal of Clinical Oncology 26.20 (2008): 3403-3410.

17. Figlin, R. A., et al. "Overall survival with sunitinib versus interferon (IFN)-alfa as first-line treatment of metastatic renal cell carcinoma (mRCC)." Journal of Clinical Oncology 26.15_suppl (2008): 50245024.

18. Gore, M. E., et al. "Sunitinib in metastatic renal cell carcinoma (mRCC): preliminary assessment of toxicity in an expanded access trial with subpopulation analysis." Journal of Clinical Oncology 25.18_suppl (2007): 5010-5010.

19. Barrett, Tanya, et al. "NCBI GEO: archive for functional genomics data sets-update." Nucleic acids research 41.D1 (2012): D991-D995.

20. Ihaka, Ross, and Robert Gentleman. "R: a language for data analysis and graphics." Journal of computational and graphical statistics 5.3 (1996): 299-314.

21. Robinson, Mark D., Davis J. McCarthy, and Gordon K. Smyth. "edgeR: a Bioconductor package for differential expression analysis of digital gene expression data." Bioinformatics 26.1 (2010): 139140. 
22. Du, Zhou, et al. "agriGO: a GO analysis toolkit for the agricultural community." Nucleic acids research 38.suppl_2 (2010): W64-W70.

23. Dennis, Glynn, et al. "DAVID: database for annotation, visualization, and integrated discovery." Genome biology 4.9 (2003): R60.

24. Moriya, Yuki, et al. "KAAS: an automatic genome annotation and pathway reconstruction server." Nucleic acids research 35.suppl_2 (2007): W182-W185.

25. Langfelder, Peter, and Steve Horvath. "WGCNA: an R package for weighted correlation network analysis." BMC bioinformatics 9.1 (2008): 559.

26. Shannon, Paul, et al. "Cytoscape: a software environment for integrated models of biomolecular interaction networks." Genome research 13.11 (2003): 2498-2504.

27. Hsu, Sheng-Da, et al. "miRTarBase: a database curates experimentally validated microRNA-target interactions." Nucleic acids research 39.suppl_1 (2010): D163-D169.

28. Wickham, Hadley. ggplot2: elegant graphics for data analysis. Springer, 2016.

29. Wickham, Hadley, and Maintainer Hadley Wickham. "Package 'plyr'." Obtenido de https://cran. rproject. org/web/packages/dplyr/dplyr. pdf (2016).

30. Steel, John R. "Scales in L (R)." Cabal Seminar 79-81. Springer, Berlin, Heidelberg, 1983.

31. Vu, Vincent Q. "ggbiplot: A ggplot2 based biplot." R package version 0.55 (2011).

32. Oksanen, Jari, et al. "The vegan package." Community ecology package 10 (2007): 631-637.

33. Chow, Tsz-fung F., et al. "The miR-17-92 cluster is over expressed in and has an oncogenic effect on renal cell carcinoma." The Journal of urology 183.2 (2010): 743-751.

34. Yamaguchi, Noriya, et al. "Identification of microRNAs involved in resistance to sunitinib in renal cell carcinoma cells." Anticancer research 37.6 (2017): 2985-2992.

35. Gámez-Pozo, Angelo, et al. "MicroRNA expression profiling of peripheral blood samples predicts resistance to first-line sunitinib in advanced renal cell carcinoma patients." Neoplasia (New York, NY) 14.12 (2012): 1144.

36. Surendran, Sankar, Stephen K. Tyring, and Reuben Matalon. "Expression of calpastatin, minopontin, NIPSNAP1, rabaptin-5 and neuronatin in the phenylketonuria (PKU) mouse brain: Possible role on cognitive defect seen in PKU." Neurochemistry international 46.8 (2005): 595-599.

37. Okuda-Ashitaka, Emiko, et al. "Identification of NIPSNAP1 as a nocistatin-interacting protein involving pain transmission." Journal of Biological Chemistry 287.13 (2012): 10403-10413.

38. Nautiyal, Manisha, et al. "Neuronal localization of the mitochondrial protein NIPSNAP1 in rat nervous system." European Journal of Neuroscience 32.4 (2010): 560-569.

39. Malhotra, Ankit, et al. "Chromosomal structural variations during progression of a prostate epithelial cell line to a malignant metastatic state inactivate the NF2, NIPSNAP1, UGT2B17, and LPIN2 genes." Cancer biology \& therapy 14.9 (2013): 840-852.

40. Khaghanzadeh, Narges, et al. "Immune-associated proteins with potential in vivo anti-tumor activities are upregulated in lung cancer cells treated with umbelliprenin: A proteomic approach." Oncology 
letters 12.6 (2016): 5295-5302.

41. Okolicsanyi, Rachel K., et al. "Heparan sulfate proteoglycans and human breast cancer epithelial cell tumorigenicity." Journal of cellular biochemistry 115.5 (2014): 967-976.

42. Dziadziuszko, Rafal, et al. "An activating KIT mutation induces crizotinib resistance in ROS1-positive lung cancer." Journal of Thoracic Oncology 11.8 (2016): 1273-1281.

43. Hakomori S .. New wine in an old bottle. Proc Natl Acad Sci (2002) USA 99: 10231-10233.

44. Chang, Shuai, et al. "MicroRNA-125b promotes invasion and metastasis of gastric cancer by targeting STARD13 and NEU1." Tumor Biology 37.9 (2016): 12141-12151.

45. Li, Dawei, et al. "The critical role of dysregulated FOXM1-PLAUR signaling in human colon cancer progression and metastasis." Clinical cancer research 19.1 (2013): 62-72.

46. Kojima, T., et al. "Decreased expression of CXXC4 promotes a malignant phenotype in renal cell carcinoma by activating Wnt signaling." Oncogene 28.2 (2009): 297.

47. Jian Zhou, et al. "PLAUR confers resistance to gefitinib through EGFR/P-AKT/Survivin signaling pathway." Cellular Physiology and Biochemistry 47.5 (2018): 1909-1924.

\section{Tables}

Table 1 Statistics of the top $10 \mathrm{GO}$ terms. 


\begin{tabular}{|c|c|c|c|}
\hline GO ID & GO Term & Ontology & GeneRatio \\
\hline G0:0008150 & biological_process & $\mathrm{BP}$ & 28 \\
\hline GO:0009987 & cellular process & $\mathrm{BP}$ & 26 \\
\hline G0:0044699 & single-organism process & $\mathrm{BP}$ & 26 \\
\hline GO:0044763 & single-organism cellular process & $\mathrm{BP}$ & 26 \\
\hline G0:0008152 & metabolic process & $\mathrm{BP}$ & 22 \\
\hline G0:0044237 & cellular metabolic process & $\mathrm{BP}$ & 21 \\
\hline G0:0044710 & single-organism metabolic process & $\mathrm{BP}$ & 21 \\
\hline G0:0044238 & primary metabolic process & $\mathrm{BP}$ & 20 \\
\hline GO:0071704 & organic substance metabolic process & $\mathrm{BP}$ & 20 \\
\hline GO:0065007 & biological regulation & $\mathrm{BP}$ & 18 \\
\hline GO:0003674 & molecular_function & MF & 30 \\
\hline G0:0005488 & binding & MF & 25 \\
\hline G0:0003824 & catalytic activity & MF & 20 \\
\hline GO:0005515 & protein binding & MF & 20 \\
\hline G0:0043167 & ion binding & MF & 13 \\
\hline GO:0016740 & transferase activity & MF & 10 \\
\hline G0:0043169 & cation binding & MF & 9 \\
\hline GO:0046872 & metal ion binding & MF & 9 \\
\hline GO:0016787 & hydrolase activity & MF & 7 \\
\hline GO:0036094 & small molecule binding & MF & 6 \\
\hline GO:0005575 & cellular_component & $\mathrm{CC}$ & 34 \\
\hline GO:0005623 & cell & $\mathrm{CC}$ & 32 \\
\hline G0:0044464 & cell part & $\mathrm{CC}$ & 32 \\
\hline GO:0005622 & intracellular & $\mathrm{CC}$ & 30 \\
\hline G0:0044424 & intracellular part & $\mathrm{CC}$ & 29 \\
\hline G0:0043226 & organelle & $\mathrm{CC}$ & 27 \\
\hline G0:0043227 & membrane-bounded organelle & $\mathrm{CC}$ & 27 \\
\hline GO:0005737 & cytoplasm & $\mathrm{CC}$ & 26 \\
\hline
\end{tabular}




\begin{tabular}{|llll|} 
GO:0043229 & intracellular organelle & CC & 24 \\
\hline G0:0043231 & intracellular membrane-bounded organelle & CC & 24 \\
\hline
\end{tabular}

Table 2 MicroRNA target gene validated by experiments.

\begin{tabular}{|ll|}
\hline MicroRNA & Target gene \\
\hline $\begin{array}{l}\text { hsa-miR- } \\
589\end{array}$ & FXN PTGS2 MAP3K8 \\
\hline $\begin{array}{l}\text { hsa-miR- } \\
\text { 18b }\end{array}$ & ESR1 MDM2 CTGF TNRC6B HIF1A SMAD2 FOXN1 IGF1 \\
$\begin{array}{l}\text { hsa-miR- } \\
30 \mathrm{e}\end{array}$ & UBE2I MYBL2 SNAI1 BMI1 MTTP MBNL1 MBNL2 NOTCH1 TP53 CASP3 MBNL3 \\
$\begin{array}{l}\text { hsa-miR- } \\
126\end{array}$ & SPRED1 PLK2 SLC45A3 CCNE2 RGS3.. \\
$\begin{array}{l}\text { hsa-miR- } \\
148 \mathrm{~b}\end{array}$ & HLA-G CCKBR ITGA5 ROCK1 PIK3CA NRAS CSF1.. \\
$\begin{array}{l}\text { hsa-miR- } \\
30 \mathrm{a}\end{array}$ & BDNF NOTCH1 BECN1 TNRC6A NT5E MBNL1 SMAD1 DTL SNAI1.. \\
$\begin{array}{l}\text { hsa-miR- } \\
708\end{array}$ & BMI1 ZEB2 BIRC5 AKT2 CD44 TMEM88 EYA3 NNAT AKT1 CCND1 MMP2 EZH2.. \\
\hline $\begin{array}{l}\text { hsa-miR- } \\
938\end{array}$ & * \\
\hline
\end{tabular}

\section{Figures}

a

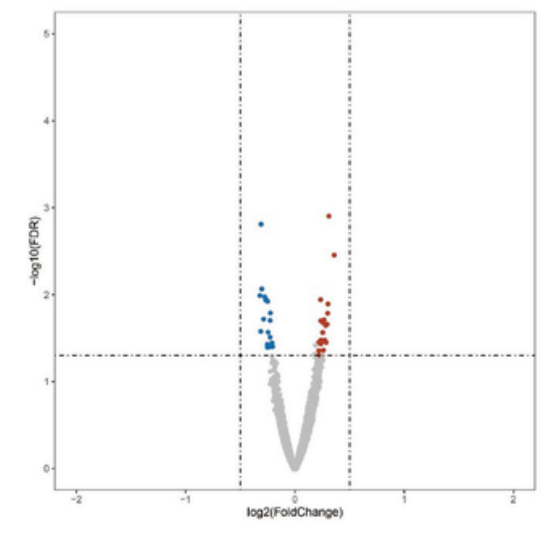

b

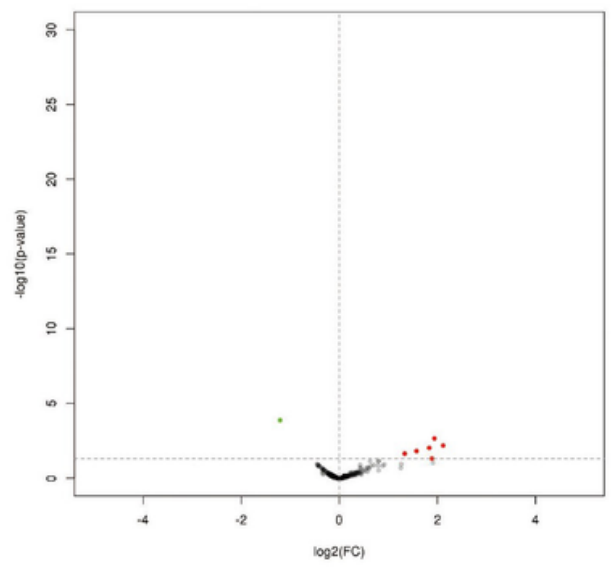

C

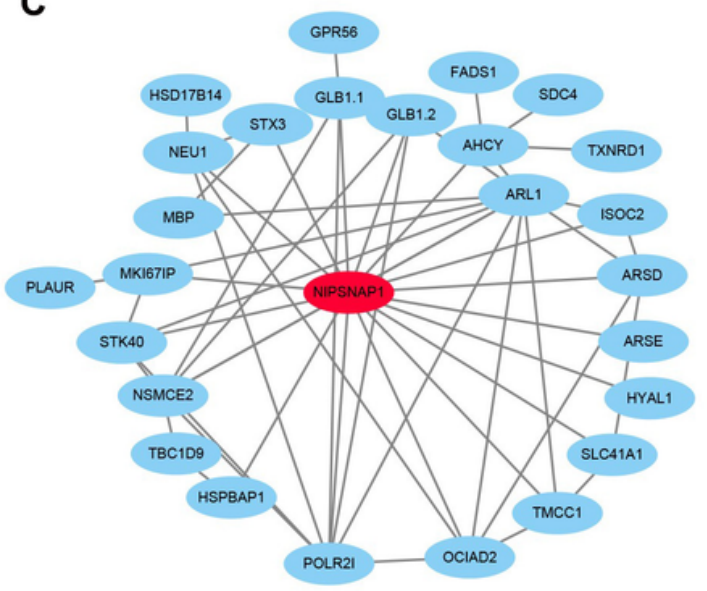

Figure 1 
Volcano figure of the differently expressed mRNA (a) and miRNA (b) $(|\log F C|>1$, adjust. $P<0.05)$. The red dots represent patients with sunitinib treatment, meanwhile, the green or blue dots represent patients without sunitinib treatment. Weighted correlation network of the differently expressed genes in turquoise module. $X$-axis represented the weighting power and $y$-axis represented the quadratic correlation index derived from $\log (k)$ and $\log (P(k))$ of the corresponding network (c).
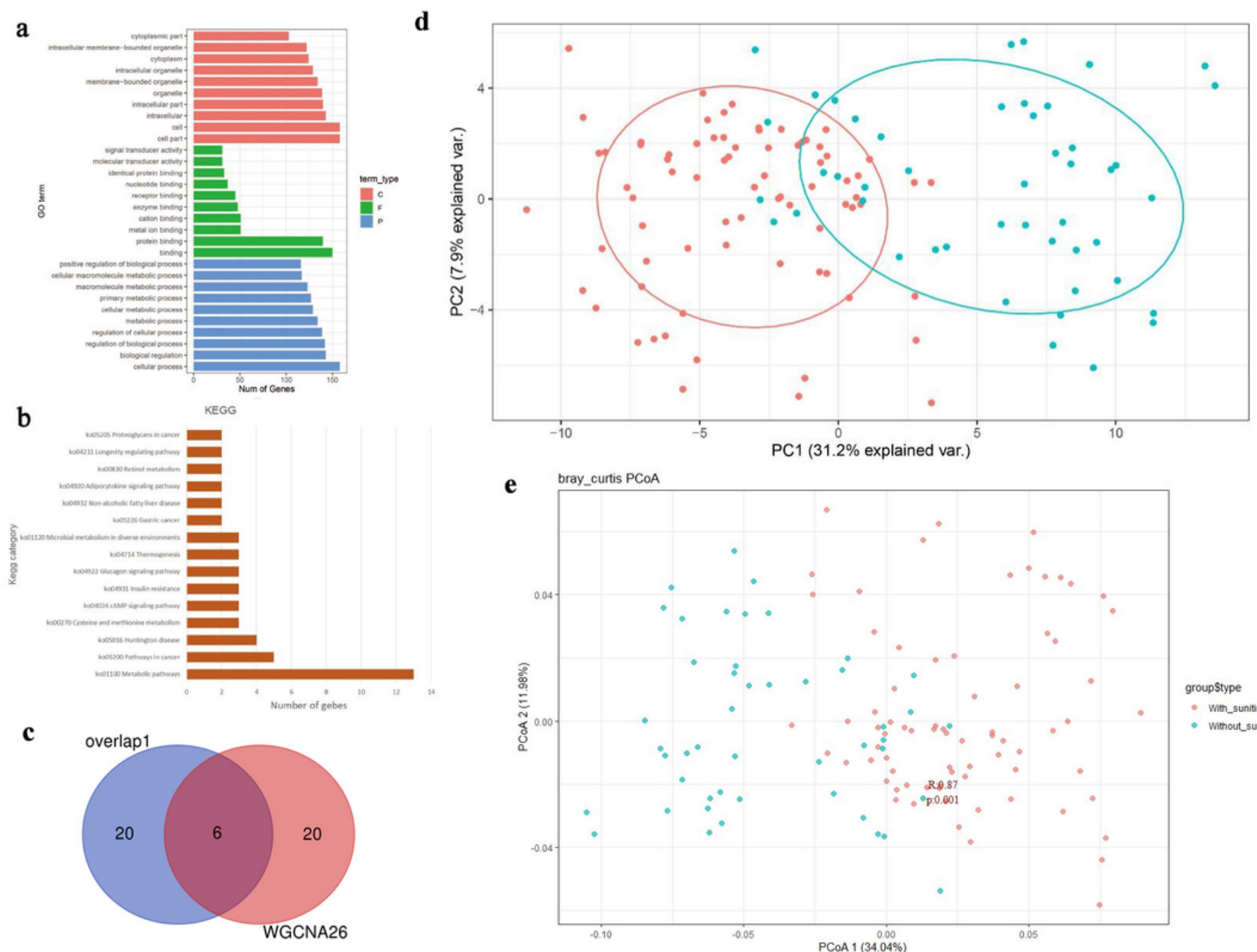

groups

$\rightarrow$ With_sunitinib

$\rightarrow$ Without_sunitinib

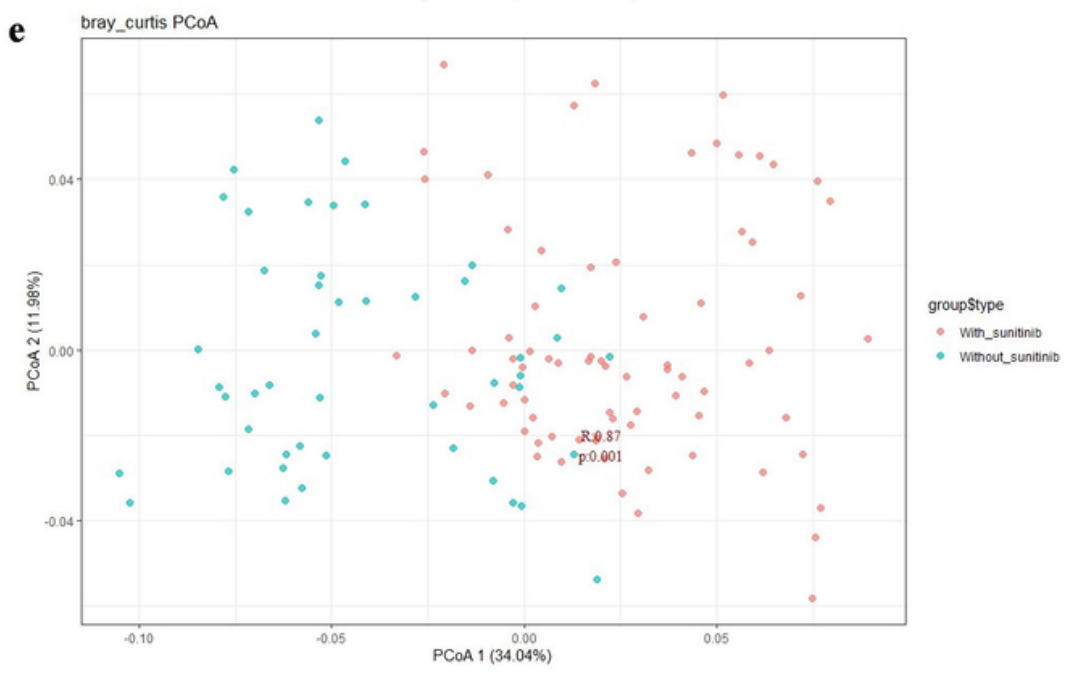

Figure 2

GO annotation of the 26 differently expressed genes in level 2 (a) and KEGG analysis of the 26 differently expressed genes (b). 26 genes were intersected with the genes in WGCNA (c). Principal component analysis (PCA) based on the 112 differently expressed genes. The $X$-axis represents the percentage of the first principal component to the total variation. The $Y$-axis represents the percentage of the second principal component in the total variation (d). Principal co-ordinates analysis (PCoA) based on the 112 differently expressed genes. $\mathrm{X}$-axis and $\mathrm{Y}$-axis of principal co-ordinates analysis represent the first principal co-ordinates and the second co-ordinates component to the total variation, respectively (e). 
NIPSNAP1

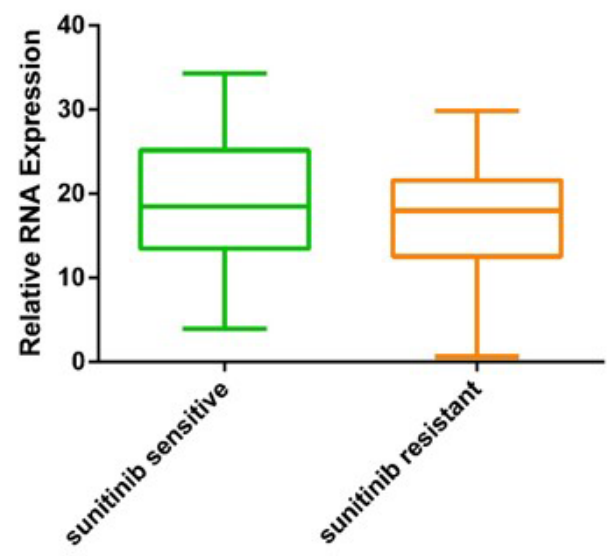

NEU1

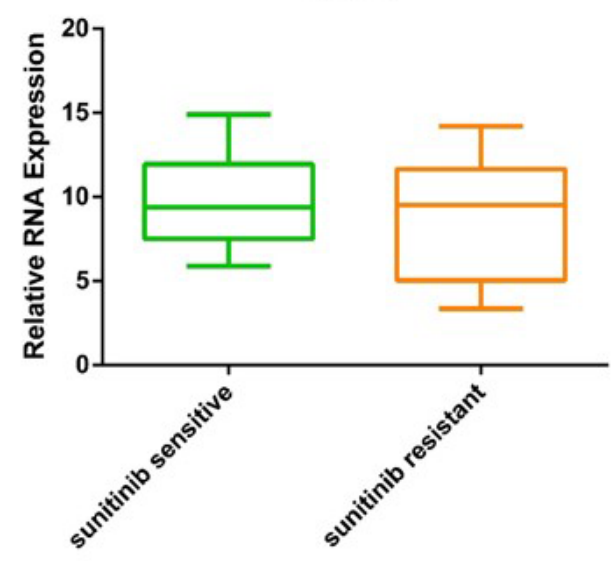

SDC4

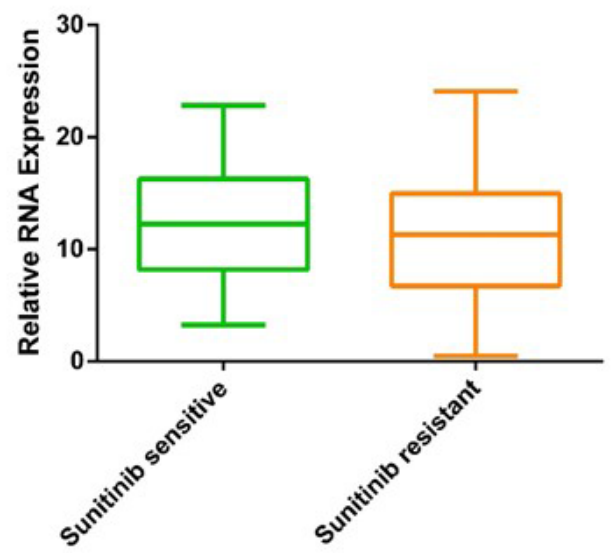

STK40

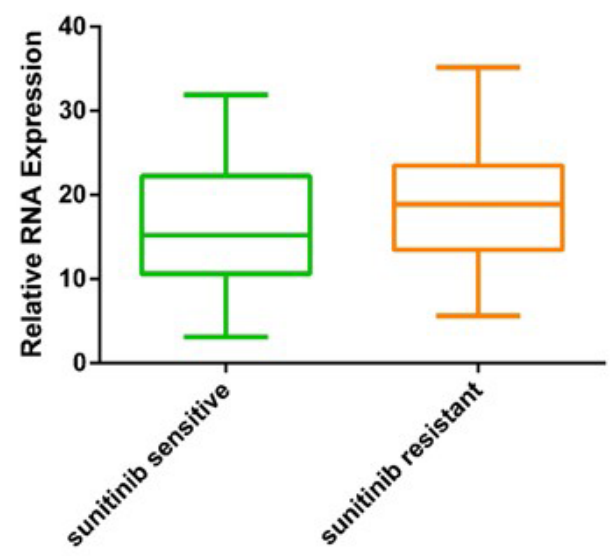

TBC1D9

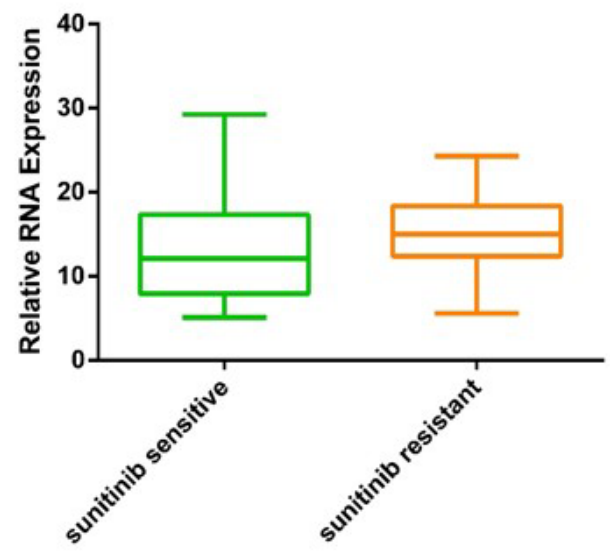

PLAUR

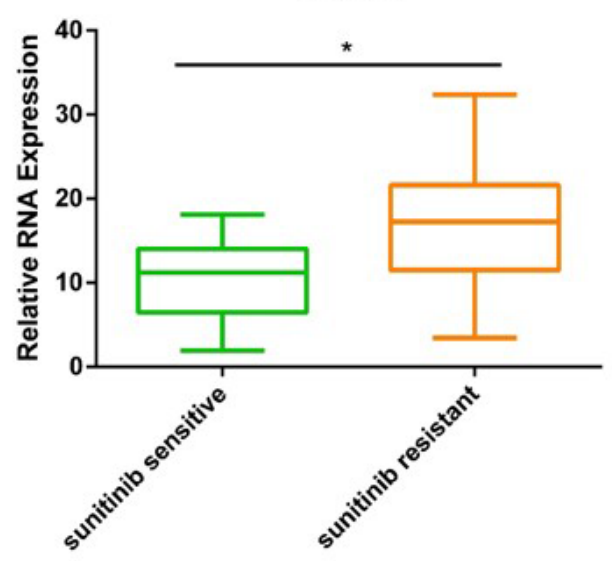

Figure 3

The six hub genes were verified by qRT-PCR experiments in sunitinib resistant and sensitive mRCC tissues. The PLAUR expression levels were consistent with the previous data obtained from the bioinformatics analysis 

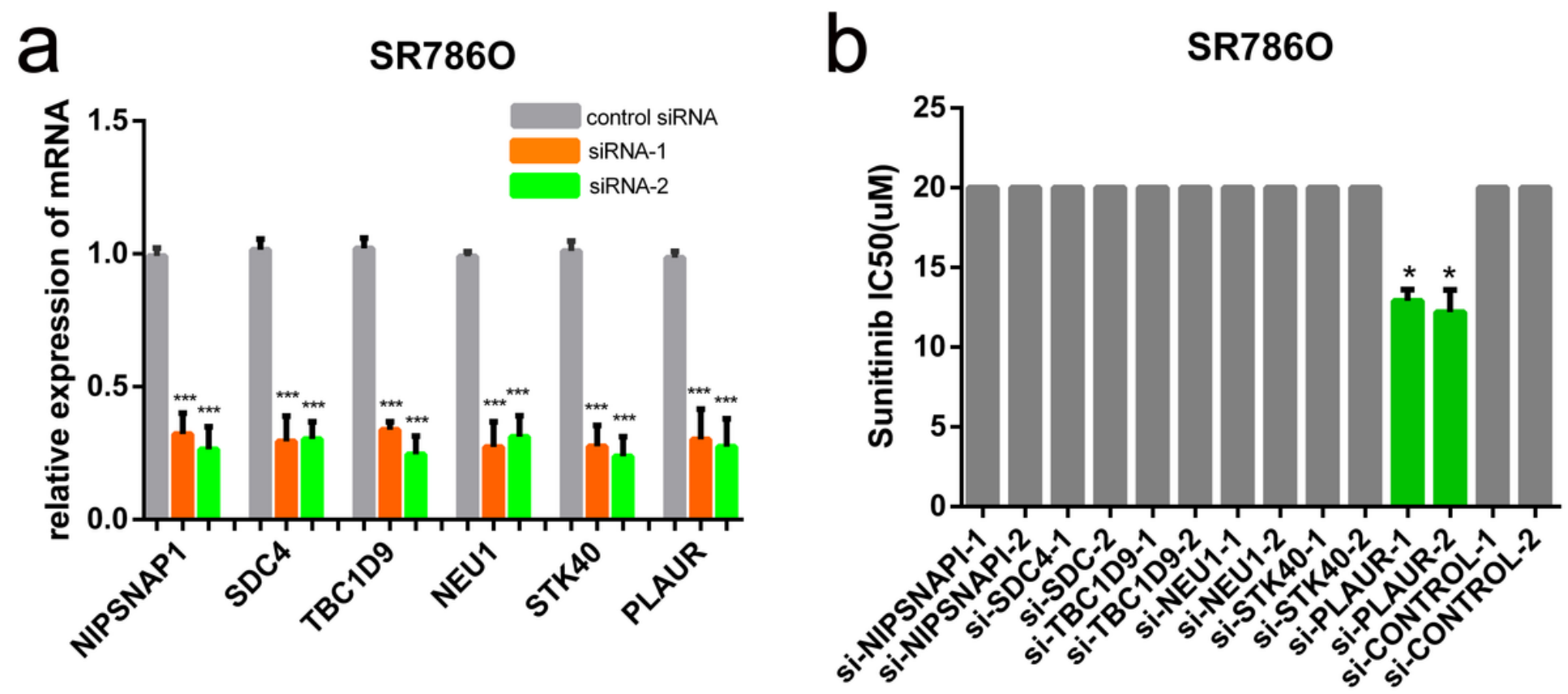

Figure 4

Validation of interference effectiveness of siRNA for indicated mRNAs in SR7860 cells by qRT-PCR $(n=3)$ (a). Detection of sunitinib IC50 in SR7860 cells downregulated with corresponding siRNAs for 60 hours $(n=3)(b)$.

Figure 5

qRT-PCR analysis on the PLAUR mRNA expression in PLAUR downregulated and control SR7860 cells $(n=3)(a)$. CCK8 experiment on the vitality of SR7860 cells incubated with indicated concentration of sunitinib $(n=4)(b)$. Microphotograph of SR7860 and transfected with shPLAUR-1/-2 SR7860 cells treated with $2 \mu \mathrm{mol}$ sunitinib for 60 hours $(\mathrm{n}=3)(\mathrm{c})$. 
a

ShCONTROL

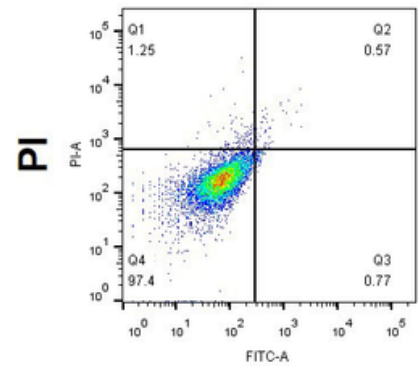

Annexin V - FITC

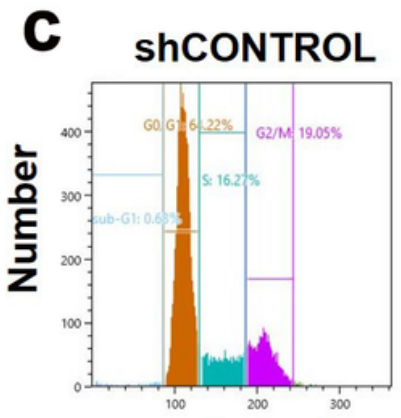

Channels
shPLAUR-1

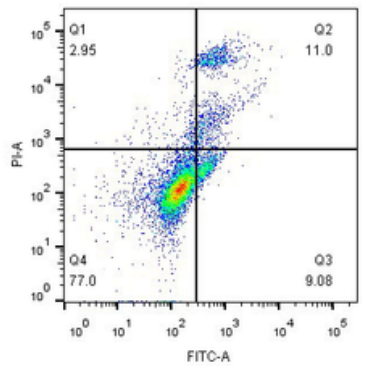

Annexin V - FITC

ShPLAUR-1

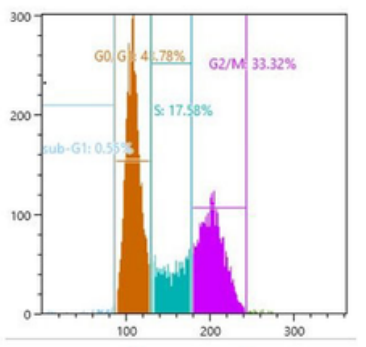

Channels
shPLAUR-2

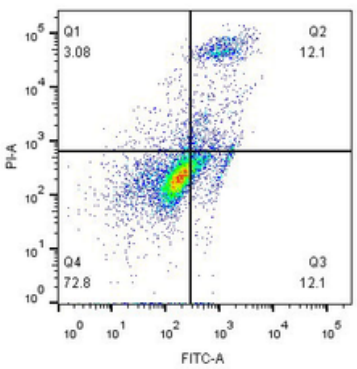

Annexin V - FITC

ShPLAUR-2

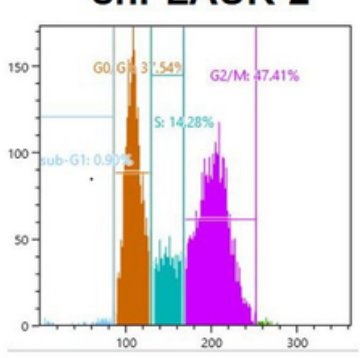

Channels b

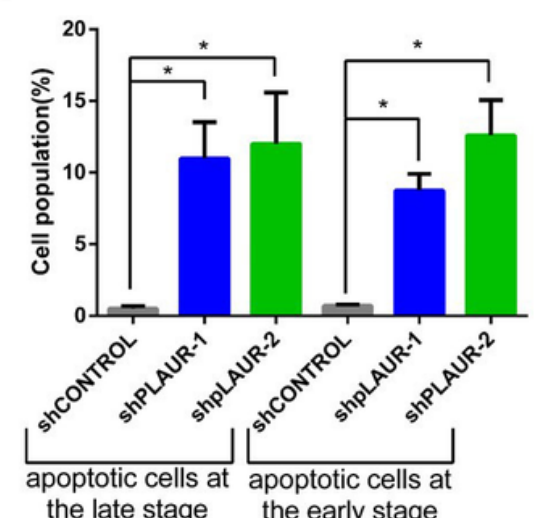

d

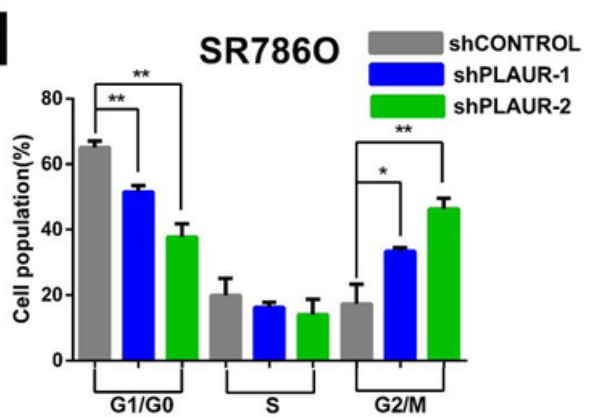

Figure 6

Annexin V-FITC/PI double dyeing experiment and flow cytometric analysis on SR7860 cells and transfected with shPLAUR-1/-2 SR7860 cells incubated with $2 \mu$ mol sunitinib $(n=3)(a)$. Quantification of apoptotic cells (\%) at the late or early stage (b). Flow cytometric analysis on cell cycle of SR7860 and transfected with shPLAUR-1/-2 SR7860 cells incubated with $2 \mu$ mol sunitinib $(n=3)(c)$. Quantification of SR7860 cell quantities at G0/G1, S and G2/M cell cycles (d). 
a

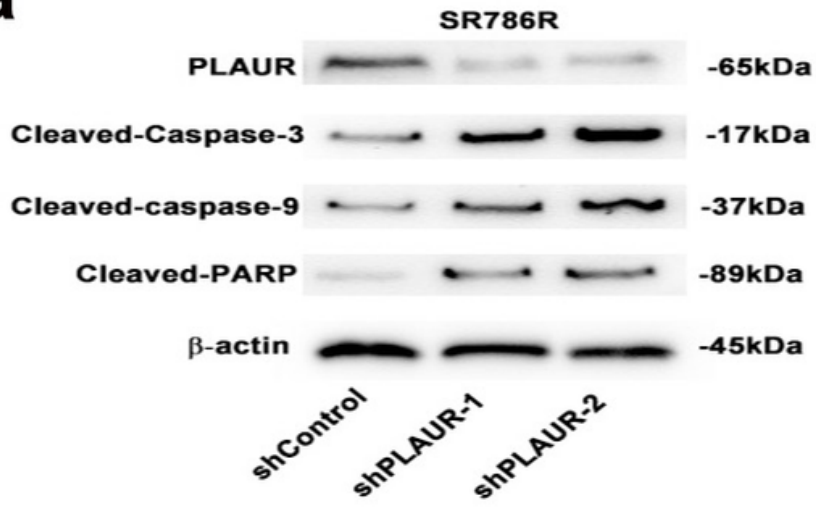

b

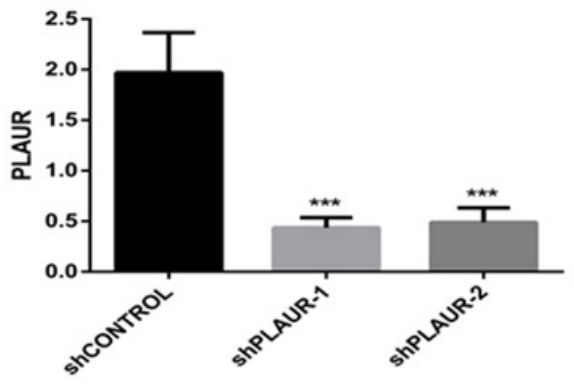

e

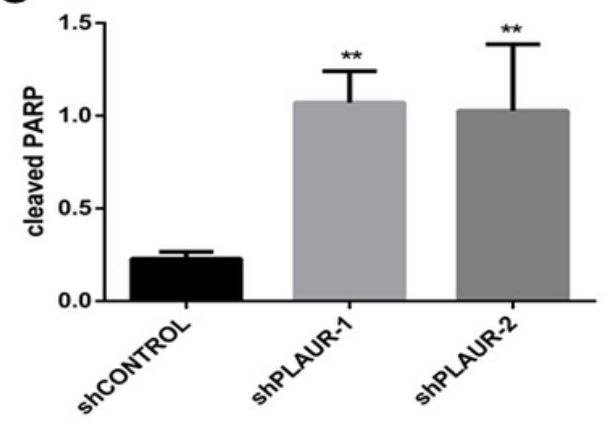

Figure 7

Western blot on total PLAUR, Cleaved-caspase-3, Cleaved-caspase-9, Cleaved-PARP (a). Quantification of Western blot PLAUR (b), Cleaved-caspase-3 (c), Cleaved-caspase-9 (d) and Cleaved-PARP (e) were normalized to $\beta$-actin $(n=3)$. 